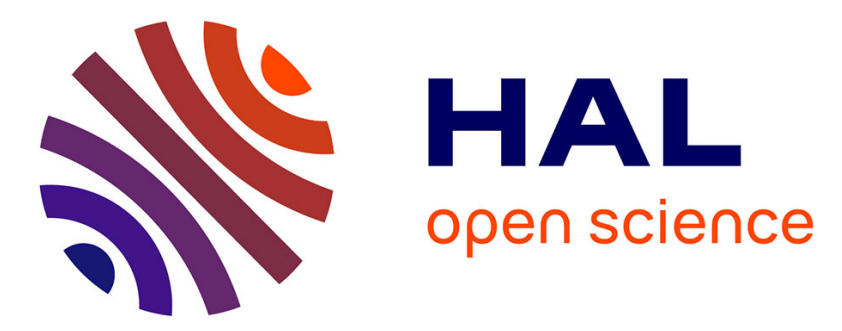

\title{
Interfacial rheological properties of self-assembling biopolymer microcapsules
}

Kaili Xie, Clement de Loubens, Frédéric Dubreuil, Deniz Gunes, Marc Jaeger, Marc Leonetti

\section{- To cite this version:}

Kaili Xie, Clement de Loubens, Frédéric Dubreuil, Deniz Gunes, Marc Jaeger, et al.. Interfacial rheological properties of self-assembling biopolymer microcapsules. Soft Matter, 2017, 13 (36), pp.62086217. 10.1039/C7SM01377A . hal-02020103

\section{HAL Id: hal-02020103 https://hal.science/hal-02020103}

Submitted on 15 Feb 2019

HAL is a multi-disciplinary open access archive for the deposit and dissemination of scientific research documents, whether they are published or not. The documents may come from teaching and research institutions in France or abroad, or from public or private research centers.
L'archive ouverte pluridisciplinaire HAL, est destinée au dépôt et à la diffusion de documents scientifiques de niveau recherche, publiés ou non, émanant des établissements d'enseignement et de recherche français ou étrangers, des laboratoires publics ou privés. 


\title{
Journal Name
}

\section{ARTICLE TYPE}

Cite this: DOI: 10.1039/xxxxxxxxxx

\section{Interfacial rheological properties of self-assembling biopolymer microcapsules ${ }^{\dagger}$}

\author{
Kaili Xie, ${ }^{a, b, c, d}$ Clément de Loubens, ${ }^{* c, d}$ Frédéric Dubreuil, ${ }^{e, f}$ Deniz Z. Gunes, ${ }^{g}$ Marc \\ Jaeger, ${ }^{a}$ and Marc Léonetti, ${ }^{* b}$
}

\section{Received Date \\ Accepted Date}

DOI: $10.1039 / x x x x x x x x x x$

www.rsc.org/journalname
Tuning the mechanical properties of microcapsules with cost-efficient route of fabrication is still a challenge. The traditional method of layer-by-layer assembly of microcapsules allows building a tailored composite multi-layer membrane but is technically complex as it requires numerous steps. The objective of this article is to characterize the interfacial rheological properties of selfassembling biopolymer microcapsules that were obtained in one single facile step. This thorough study provides new insights in the mechanics of these weakly cohesive membranes. Firstly, suspensions of water-in-oil microcapsules were formed in microfluidic junctions by self-assembling of two oppositely charged polyelectrolytes, namely chitosan (water soluble) and phosphatidic fatty acid (oil soluble). In this way, composite membranes of tunable thickness (between 40 - 900 $\mathrm{nm}$ measured by AFM) were formed at water / oil interfaces in a single step by changing the composition. Secondly, microcapsules were mechanically characterized by stretching them up to break-up in an extensional flow chamber which extends the relevance and convenience of the hydrodynamic method to weakly cohesive membranes. Finally, we show that the design of microcapsules can be 'engineered' in a large way since they present a wealth of interfacial rheological properties in term of elasticity, plasticity and yield stress whose magnitudes can be controlled by the composition. These behaviors are explained by the variation of the membrane thickness with the physico-chemical parameters of the process.

\section{Introduction}

Microcapsules are drops surrounded by thin elastic shells that have the ability to protect and deliver substances in biological or industrial processes. They have a great potential for the development of new multi-functional materials as paints ${ }^{1}$ but also to deliver nutrients, bacteria, DNA or drugs in the human body via the blood microcirculation ${ }^{2,3}$ or the digestive tract ${ }^{4-6}$. Controlling the mechanical properties of microcapsules is of prime importance to provide their stability in various physical and chemical

\footnotetext{
${ }^{a}$ Aix-Marseille Université, CNRS, Centrale Marseille, M2P2 UMR 7340, 13451, Marseille, France.

${ }^{b}$ Aix-Marseille Université, CNRS, Centrale Marseille, IRPHE UMR 7342, 13384, Marseille, France.E-mail: leonetti@irphe.univ-mrs.fr

${ }^{c}$ Université Grenoble Alpes, LRP, F-38000 Grenoble, France.

${ }^{d}$ CNRS, LRP, F-38000 Grenoble,France.E-mail: clement.de-loubens@univ-grenoblealpes.fr

e Université Grenoble Alpes, CERMAV, F-38000 Grenoble, France.

${ }^{f}$ CNRS, CERMAV, F-38000 Grenoble, France.

${ }^{g}$ Nestlé Research Center, Vers-chez-les-Blanc, PO Box 44, 1000 Lausanne 26, Switzerland.

$\dagger$ Electronic Supplementary Information (ESI) available: [details of any supplementary information available should be included here]. See DOI: 10.1039/b000000x/
}

environnement and to release encapsulated molecules ${ }^{7}$.

In this regard, layer-by-layer assembly of polyelectrolytes on dissolvable colloids is considered as a gold standard method, since it allows to control accurately the thickness of the membrane ${ }^{8-10}$ and so its mechanical properties ${ }^{11,12}$. But the drawback of this multi-steps method is its length when a large number of layers has to be deposited to get high mechanical resistance. Recently, facile routes of fabrication of biopolymer microcapsules have been proposed that takes advantage of the self-assembling of polylectrolytes at water and oil interfaces ${ }^{13-16}$. As example, polymer - surfactant complex has been used to encapsulate Vitamine $\mathrm{E}$ in oil droplets ${ }^{17}$. A biocompatible solution has been obtained by the complexation of chitosan, a water-soluble selfhealing biopolymer ${ }^{18}$, with a counter-charged oil-soluble ionic surfactant, in order to form membranes at the interfaces of microdroplets $^{18}$. Chitosan ${ }^{19}$ acting as a kind of polysaccharide carrying positively charged groups (Figure 1) presents good solubility in water under acid condition (e.g., $\mathrm{pH}<4$ ) with a great potential in drug delivery due to its biocompatibility and mucoadhesive porperties ${ }^{6}$. The surfactant phosphatidic fatty acid is a type of food molecule well-known in chocolate manufacture, as well as 
a component of cell membranes ${ }^{18}$, referred to as 'PFacidYN', exhibiting negative charges (Figure 1), which is insoluble in water but well soluble in vegetable oils. The membrane obtained with these complexing molecules has a multi-layer aspect with a disordered structure and its thickness grows by a diffusion-controlled mechanism ${ }^{18}$, hence can be tuned by varying concentrations of complexing molecules and complexation time. However the robustness of this method in terms of stability and control of the mechanical properties of the microcapsules is unknown while it is the main condition for its scale-up. Indeed the resulting mechanical properties of these kind of self-assembling biopolymer microcapsules have not been characterized in a large range of deformations and conditions of fabrication.

Mechanical properties of thin membrane of microcapsules are described generally by bi-dimensional non-linear viscoelastic constitutive models ${ }^{20-22}$, as for fluid interfaces ${ }^{23,24}$. Rheology of complex plane fluid-fluid interfaces can be characterized by specific tools that respond to small surface stresses in isolating them from bulk stresses as the double-wall ring geometry rheometer ${ }^{25}$. In contrast, the linear elastic properties of solid membrane of microcapsules can be inferred from direct experiments on microcapsules ${ }^{26}$, for example osmotic pressure tests ${ }^{27-29}$, atomic force microscopy (AFM) ${ }^{11,30}$ or the analysis of wrinkles on deflated microcapsules ${ }^{31}$. Whereas advanced tools of interfacial rheology tend to dissociate the mechanical response of fluid-fluid interfaces from the fluid mechanics of the bulk phases ${ }^{25}$, Chang \& Olbricht ${ }^{32,33}$ have demonstrated the strength of hydrodynamics methods to deform millimetric capsules with simple flows and to determine surface elastic modulus. More recently, these methods have been extended to micrometric capsules ${ }^{34-38}$. By combination with advanced numerical simulations ${ }^{21,22,39}$, the nonlinear elastic constitutive laws in regime of large deformation ${ }^{40}$ and the viscosity of the membrane ${ }^{41}$ have been determined for microcapsules made from a biopolymer (HSA) that was physically cross-linked to form a thin membrane in hydrogel. It was shown that this kind of microcapsules can sustained up to $180 \%$ of stretching without plastic deformations or break-up. If microfluidic hydrodynamic stretching has been proved relevant and convenient to determine the mechanical properties of strongly cohesive membranes such as made with HSA, the method has never been applied to weakly cohesive membranes such as multilayers of polyelectrolytes. Mechanical properties of such microcapsules have mainly been characterized in small deformation regime by osmotic pressure tests, micromanipulation ${ }^{12}$ or by applying stress / strain on plane surfaces ${ }^{16,18}$ showing viscoelastic and self-healing properties of this kind of self-assembling membranes. Here we will demonstrate the advantage of hydrodynamic methods, namely the elongation flow, to characterize as well the linear as the non-linear interfacial rheological properties of polyelectrolytes chitosan / PFacidYN microcapsules. Moreover, we will show that the mechanical behaviour of this kind of polyelectrolyte microcapsules differ greatly from those of cross-linked biopolymer membranes.

The objective of this paper is to characterize the interfacial rheological properties of self-assembling biopolymer microcapsules that were obtained in one single facile step. The aim is to

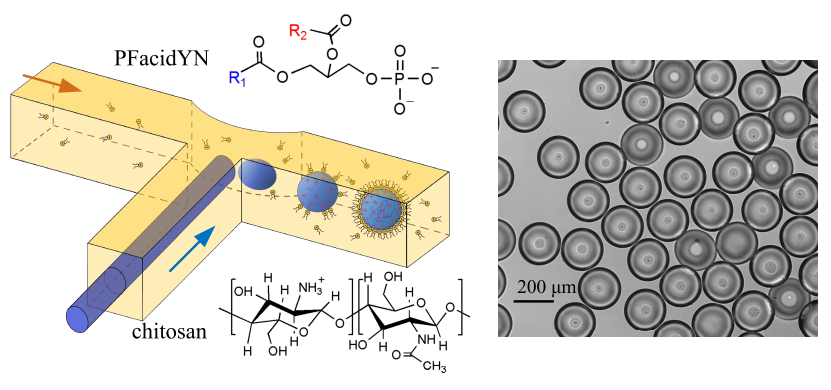

Fig. 1 Fabrication of monodisperse chitosan / PFacidYN microcapsules. Left: Formation of aqueous droplets of chitosan dispersed in an oil phase containing the surfactant PFacidYN in a T-junction. The shell is formed around an aqueous phase droplet by electrostatic interactions between chitosan and PFacidYN molecules. Right: Optical micrograph of monodisperse chitosan / PFacidYN microcapsules.

question their fabrication robustness and provide new insights of their non-linear rheological behavior. Firstly, suspensions of chitosan / PFacidYN microcapsules ${ }^{18}$ were produced using microfluidics droplets generators with various concentrations of complexing molecules and size of droplets. We paid attention also to control the time of complexation of the two electrolytes at water / oil interface and the stability of the microcapsules in regard to osmotic shocks outside the fabrication system. Secondly, the linear and non-linear interfacial rheological properties of these microcapsules were characterized by stretching them under controlled hydrodynamic stresses in a milli-fluidic extensional flow chamber ${ }^{38,42}$. We show that surface shear elastic modulus depends on the composition and the complexation time. Moreover, the microfluidic fabrication method allowed us to get suspensions of monodisperse microcapsules with uniform mechanical properties. Finally chitosan / PFacidYN microcapsules show remarkable non-linear rheological behaviors as yield stress (i.e. solid / liquid transition) and break-up that can be tuned also via the formulation and are related to the thickness of the membrane measured by Atomic Force Microscopy (AFM). We conclude on the opportunities offered by theses results to 'engineer' in a facile route the mechanical properties of self-assembling biopolymer microcapsules for specific applications.

\section{Materials and methods}

\subsection{Chitosan solutions}

Chitosan (CAS number 9012-76-4, Sigma-Aldrich) with medium molecular weight and 75-85\% deacetylation (Figure 1) was used for the fabrication of polyelectrolytes microcapsules. Chitosan powder was dissolved in Millipore water (resistivity $>18 \mathrm{M} \Omega$ $\mathrm{cm})$ by adjusting the $\mathrm{pH}$ with hydrochloric acid $(1 \mathrm{~mol} / \mathrm{L})$ at 3.0. The chitosan solution was then filtered to remove undissolved particles with syringe-filters Minisart (pore size of $5.0 \mu \mathrm{m}$ ). The density was measured with the densimeter DMA $4500 \mathrm{M}$ (Anton Paar) and adjusted to $1.09 \pm 0.01 \mathrm{~g} / \mathrm{mL}$ by addition of pure glycerol at $22^{\circ} \mathrm{C}$ (98\% of purity, CAS number 56-81-5, VWR) to avoid sedimentation or creaming in silicon oil Wacker AP1000 that was kindly offered by IMCD group. All Chitosan solutions used in this investigation were stored at $4^{\circ} \mathrm{C}$. Before microcapsules fabrication, the $\mathrm{pH}$ value of the mixture of chitosan and glycerol solution 

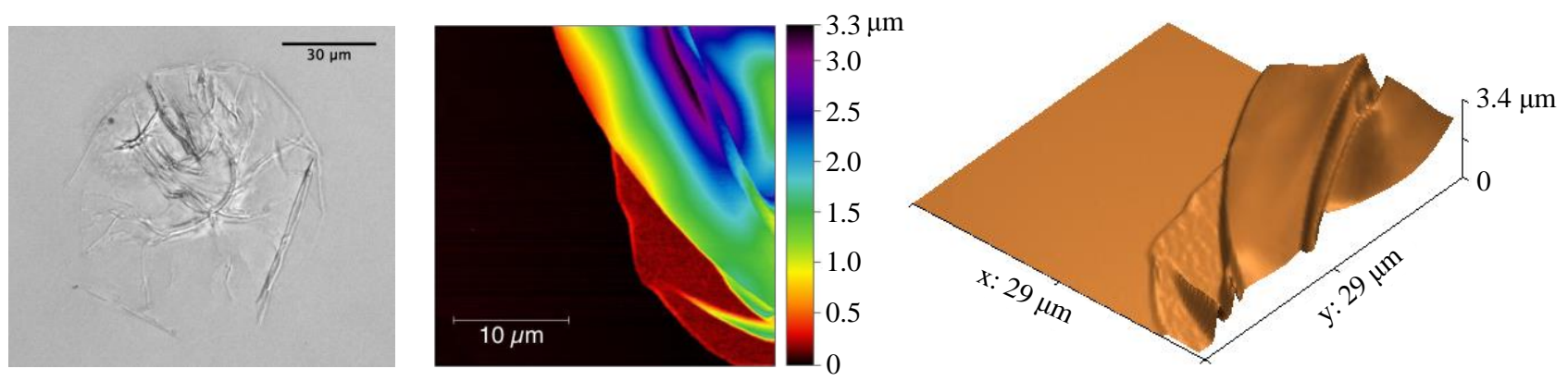

Fig. 2 Measurement of membrane thickness by AFM. Left: Optical microscopy of a dried microcapsule on a silicium wafer. The microcapsule has collapsed on itself. Middle: Topographic image of a $30 \times 30 \mu \mathrm{m}^{2}$ area of a dried microcapsule. The membrane thickness is estimated by measuring the height of the collapsed capsule flat regions. Right: Three dimensional reconstruction of the topographic image.

was checked and eventually re-adjusted at $3.00 \pm 0.02$. Table 1 in supplementary materials summarizes the different solutions of chitosan / glycerol used for microcapsules fabrication. Their viscosities range from 13.9 to $794 \mathrm{mPa} \cdot \mathrm{s}$ at $22^{\circ} \mathrm{C}$.

\subsection{Solutions of phosphatidic fatty acids in oil}

The surfactant used to precipitate capsule membrane is ammonium phosphatidic fatty acid (PFacidYN, Figure 1). It is comprised in the commercial lecithin YN (Palsgaard 4448, food-grade, E442) that was provided in kind by Palsgaard. In mass, the phosphatidic acids are 55\% w/w, neutral triglycerides $40 \%$ and ammonium salts $5 \%$, see Minifie ${ }^{43}$ and Gunes et al. ${ }^{18}$ for details. PFacidYN was dissolved in rapeseed oil (CAS number 8002-13-9, Sigma-Aldrich) at different concentrations (Table 1 in supplementary materials). Undissolved particles were removed by centrifugation. The viscosity of these solutions was about $65-70 \mathrm{mPa} \cdot \mathrm{s}$ according to the concentration of PFacidYN.

\subsection{Fabrication of monodisperse microcapsules}

Monodisperse chitosan / PFacidYN microcapsules were fabricated in a home-made microfludic T-junction (Figure 1) that produced water-in-oil droplets ${ }^{44}$. The chip was manufactured by a 3-D printer (Formlabs Inc., Form 1+) and made of a hydrophobic resin (Clear, FLGPCL03, Formlabs). A round glass capillary (from CM Scientific Ltd.) of $200 \mu \mathrm{m}$ internal diameter and $65 \mu \mathrm{m}$ thick was inserted into the side channel branch where the aqueous phase was pumped into. The intersection area in the flow direction of the continuous phase (rapeseed oil) was narrowed to strengthen the shear forces and to generate aqueous droplets. The two immiscible phases were driven by pressure and flow rate controllers (Fluigent). Consequently, we were able to produce monodisperse suspensions of spherical microcapsules: the standard deviation of the size distribution was within 4\% (Figure 1). Various microcapsules radii have been obtained by adjusting the flow rates of both phases with the same set-up: from 80 to 200 $\mu \mathrm{m}$.

To obtain uniform suspensions of microcapsules in term of interfacial rheological properties, it is necessary to control the time of membrane formation due to the complexation of chitosan with PFacidYN. Firstly, microcapsules were pre-formed in the microfluidic chip with the oil phase containing a very low concentration of PFacidYN $(<0.01 \% \mathrm{w} / \mathrm{w})$ to stabilise the droplet and avoid coalescence, whereas the aqueous phase contained the desired concentration of chitosan /glycerol mixture. These capsules were collected for 5 minutes. During this time, the membrane formation was very limited as the concentration of PFacidYN was low. Secondly, the microcapsules were transferred into an oil phase with 0.066 to $2.75 \% \mathrm{w} / \mathrm{w}$ PFacidYN. In this way, the time let for the complexation of chitosan with PFacidYN, $t_{\text {comp }}$, can be controlled easily and varied from 2 to $25 \mathrm{~min}$. Thirdly the reaction was stopped by diluting the oil phase with a large quantity of mineral oil (CAS number 8042-47-5, Sigma Aldrich) and cyclohexane (CAS number 110-82-7, assay 99.5\%, Sigma Aldrich) with a ratio 4:1 v/v. Microcapsules were let to sediment within a few minutes and the supernatant organic phase was removed. The microcapsules were dispersed in silicone oil Wacker AP1000 to be streched in elongation flow. Density of the internal fluid (chitosan / glycerol mixture) was similar to the density of the silicon oil to avoid sedimentation or creaming. The viscosity of silicone oil AP1000 was $1.18 \mathrm{~Pa} \cdot \mathrm{s}$ at $22^{\circ} \mathrm{C}$ for shear rates up to $500 \mathrm{~s}^{-1}$.

\subsection{Atomic Force Microscopy}

The membrane thickness of dried microcapsules was measured by Atomic Force Microscopy (AFM). Firstly, microcapsules deposited on a silicon wafer were left to dry for a week at $35^{\circ} \mathrm{C}$. Microcapsules were so collapsed on themselves during this process (Figure 2-left). The topology of the surface of collapsed microcapsules was done by AFM. AFM measurements were carried out with a Dimension Icon (Brucker) and a Pico Plus (Molecular Imaging) equipped with silicon probes having a nominal spring constant of 0.2 N.m ${ }^{-1}$ and tip radius below $10 \mathrm{~nm}$ (All in One AFM probes from Budget Sensors) using standard procedure for contact mode in fluids. Data were treated and analysed using Gwyddion software. Topographic variations were obtained after baseline correction via profile measurements on various microcapsules ${ }^{11}$. The membrane thickness was estimated by measuring the height of the collapsed capsule flat regions (Figure 2) and ranged from 40 to $900 \mathrm{~nm}$ according to the formulation used to fabricate the microcapsules. 

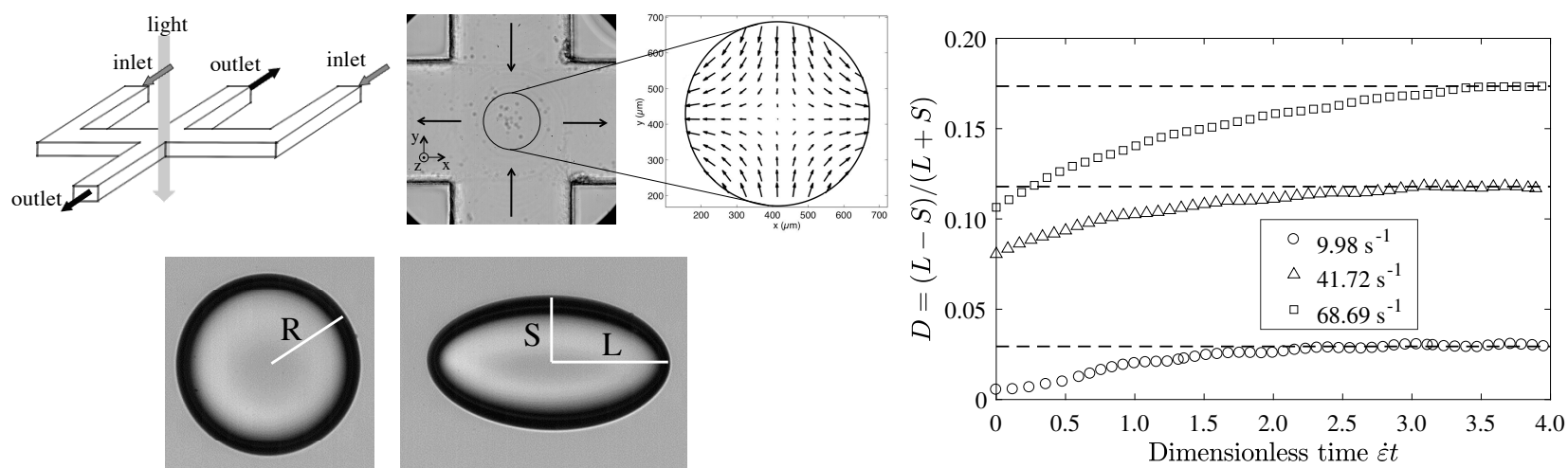

Fig. 3 Stretching of microcapsules. Left: Individual microcapsules are stretched in the center of the cross $\left(1 \times 1 \mathrm{~mm}^{2}\right)$ by an elongation flow. The deformation is characterized by the small $S$ and large $L$ axis of the microcapsule, and by the Taylor parameter $D=(L-S) /(L+S)$. Right: Temporal evolution of the Taylor parameter $D$ as a function of dimensionless time for different rates of elongation $\dot{\varepsilon}$. The steady-state deformation $D_{\infty}$ is determined as the plateau value of each curve. See Movie 1 in Supplementary Materials.

\subsection{Elongation flow chamber}

The interfacial rheological properties of microcapsules were determined by stretching individual microcapsules in an elongation flow chamber. ${ }^{38,40,42}$ Planar elongation flow was generated in a cross-like channel made of PMMA with $1 \mathrm{x} 1 \mathrm{~mm}^{2}$ square-sections (Figure 3). The fluid was injected into the chamber by a glass syringe mounted on a home-made syringe pump driven by a PI actuator M235-52S. We paid attention to avoid any deformable pipes and any air bubbles to minimize the transient time when the flow was switched on. The visualization was achieved with an inverted microscope Olympus IX-71 with a $20 \times$ magnification. A high speed video camera (Photron Fastcam SA3) enables us to acquire up to 5000 frames per second. The image resolution was $0.851 \mu \mathrm{m}$ by pixels. The temperature was controlled by a digital thermocouple with a precision of $0.1^{\circ} \mathrm{C}$. All the experiments were carried out at $22 \pm 0.5^{\circ} \mathrm{C}$.

The rate of elongation in the center of the cross was measured by Particle Tracking Velocimetry (PTV), see de Loubens et al. ${ }^{38}$ for details. A suspension of $1.6 \mathrm{mg} / \mathrm{mL}$ spherical ploystyrene particles (from Bangs Lab) with average diameter of $10 \mu \mathrm{m}$ was seeded in silicone oil AP1000. The rate of elongation was found to be uniform in a region of interest (ROI) of $250 \mu \mathrm{m}$ around the stagnation point where the flow is zero and unstable. On the center-plane $(z=0)$, the velocity field $\mathbf{v}$ is given by $\mathbf{v}=\dot{\boldsymbol{\varepsilon}}(x \mathbf{x}-y \mathbf{y})$ where $\dot{\varepsilon}$ is the rate of elongation (in $s^{-1}$ ), $\mathbf{x}$ and $\mathbf{y}$ the cartesian axis with origin the stagnation point (Figure 3 ). All capsule deformation analysis should be conducted in the ROI. Within the range of flow rate 1 to $400 \mu \mathrm{L} / \mathrm{s}$, a linear variation of the elongation rate was found. In addition, the dependence of $\dot{\varepsilon}$ on the depth of the channel (z direction) was characterized. As expected, the flow had a Poiseuille-like profile. For each measurement, the $z$ position of the microcapsule was measured to determine accurately the elongation rate using the law $\dot{\varepsilon}=f(z)$ that was determined experimentally ${ }^{38}$. To prevent any effect of the channel walls, only capsules close to the center (i.e. distance from the center below $\pm 100 \mu \mathrm{m}$ ) were studied. For radii of $200 \mu \mathrm{m}$, the capsule sees only a variations of $2 \%$ of the rate of elongation in these conditions. The projection of a deformed capsule on the $x-y$ plane was assumed to be an ellipse with the major and minor axis length $L$ and $S$, respectively. In fact, this is the first shape mode which is excited by the elongation flow whatever the nature of the soft particle at the center. We defined the Taylor parameter by

$$
D=\frac{L-S}{L+S}
$$

For low values of elongation rate $\left(\dot{\varepsilon}<30 \mathrm{~s}^{-1}\right)$, capsules were trapped one by one at the stagnation point of the elongation flow. Two containers at the ambient pressure were plugged to the outlets of the channel, which allowed to stabilize the microcapsule at the stagnation point. Then we suddenly applied the flow and measured the deformation as function of the time (Figure 3, $\dot{\varepsilon}=$ $9.98 \mathrm{~s}^{-1}$ ). The steady-state deformation $D_{\infty}$ was extracted from the plateau of the curve. Alternatively, for high values of rates of elongation $\left(\dot{\varepsilon}>30 \mathrm{~s}^{-1}\right)$, the microcapsule was sucked up in one branch of the channels up to $5 \mathrm{~mm}$ from the stagnation point and the flow was applied. The capsule came so from one of the two inlet branches of the channel, then passed by the stagnation point and went back in a perpendicular branch (outlet). In this way, it was possible to obtain a saturated dynamics and large deformations of the microcapsules (Figure 3, $\dot{\varepsilon}=41.72$ and 68.69 $\mathrm{s}^{-1}$ ) before the capsule moved out of the ROI. We checked that the two ways were equivalent for moderate rates of elongation ${ }^{40}$. The rate of elongation $\dot{\varepsilon}$ was varied from 0.5 to $300 \mathrm{~s}^{-1}$.

The hydrodynamic stress $\sigma$ (in Pa) applied on the microcapsule in the center of the cross-like channel is

$$
\sigma=\eta \dot{\varepsilon}
$$

where $\eta$ is the viscosity of the external phase, i.e. the silicone oil AP1000. For microcapsules with thin membranes, their interfacial rheological properties can be modelled by surface constitutive laws without bending resistance. Various constitutive laws ${ }^{21,39}$ describe the elastic deformation of microcapsules in small and large deformations: generalized Hooke law, Skalak law, neo-Hookean law. In the limit of small deformations, all these laws reduced to the linear Hooke law with the same surface shear elastic modulus $G_{s}$ (in $\mathrm{N} / \mathrm{m}$ ) that is related to the stable deforma- 


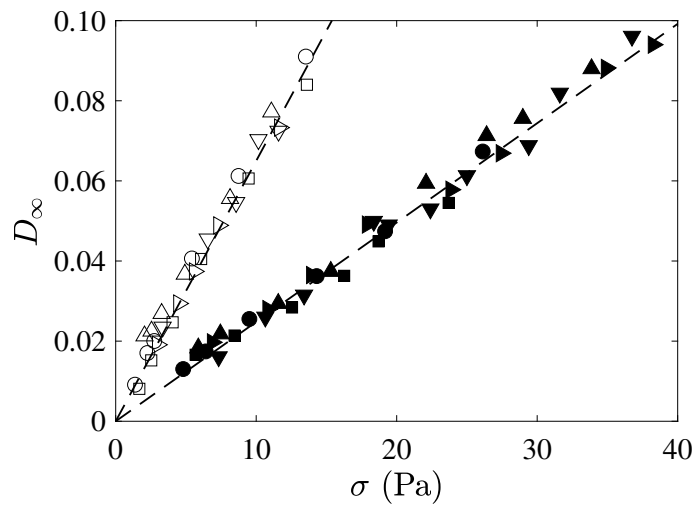

Fig. 4 The steady-state Taylor parameter $D_{\infty}$ increases linearly with the hydrodynamic stress $\sigma$ for two different formulations of microcapsules. Each symbol corresponds to a microcapsule. The surface shear elastic modulus $G_{s}$ is determined from the slope of the curve for each microcapsule (dotted lines, Equation 3). White symbols: $0.25 \% \mathrm{w} / \mathrm{v}$ chitosan, $0.66 \%$ PFacidYN, 2 min complexation time, $102 \pm 1 \mu \mathrm{m}$ radius. Black symbols : $0.11 \% \mathrm{w} / \mathrm{v}$ chitosan, $0.36 \% \mathrm{PFacidYN}, 2 \mathrm{~min}$ complexation time, $102 \pm 1 \mu \mathrm{m}$ radius.

tion $D_{\infty}$ under elongation flow by ${ }^{45}$

$$
G_{S}=\frac{25}{6} \frac{\sigma R}{D_{\infty}}
$$

Note that $G_{s}$ can be correlated with the three dimensional Young modulus $E$, the Poisson coefficient $v$ and the thickness of membrane $h$ by

$$
G_{s}=\frac{E h}{2(1+v)}=\frac{E h}{3}
$$

if the membrane is assumed to be made of an uniform incompressible elastic material $(v=0.5)$. The determination of the surface viscous modulus of the membrane is out of the scope of this paper. This parameter is difficult to determine by experiments on microcapsules as we need to track the movement of the membrane in shear flow and deduce the viscous modulus from inverse numerical simulations ${ }^{22,41}$.

Each microcapsule was tested for at least six different values of elongation rates covering a range with at least a factor two. Nineteen different formulations of microcapsules were tested (Table 2 in supplementary materials) with 4-7 microcapsules by batch. The radius was also varied from 80 to $200 \mu \mathrm{m}$. Finally, more than 120 microcapsules in total were analyzed in this paper.

\section{Results and discussion}

\subsection{Elastic properties of microcapsules}

Our experimental set-up allowed us to apply different hydrodynamic stress $\sigma$ on a given microcapsule and to analyse the evolution of its deformation. The steady-state Taylor parameter $D_{\infty}$ varied linearly with increasing of stress $\sigma$ within the small deformation regime (Figure 4), as expected by asymptotic theories 45 $\left(D_{\infty}<0.1\right)$. In this regime, the shape of the microcapsule recovered to initially spherical status after the flow stopped. Different microcapsules with the same formulation exhibited similar behavior, which means that capsules fabricated with our present

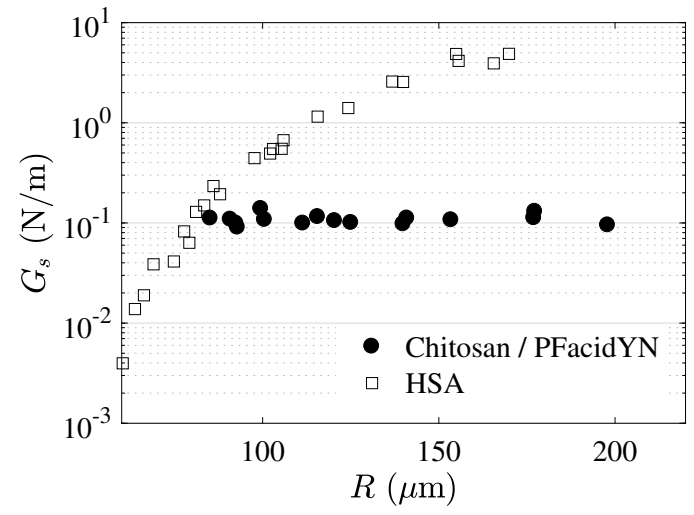

Fig. $5 G_{s}$ as a function of the radius $R: 0.25 \% \mathrm{w} / \mathrm{v}$ chitosan, $0.36 \% \mathrm{w} / \mathrm{w}$ PFacidYN, 2 min complexation time. Comparison is made with HSA microcapsules (data from de Loubens et al. ${ }^{38}, 10 \% \mathrm{w} / \mathrm{w} \mathrm{HSA}$ ). $G_{s}$ is almost constant for chitosan / PFacidYN microcapsules, whereas it varies of several orders for HSA microcapsules. Each symbol is the mean of 4 microcapsules for chitosan /PFacidYN microcapsules and represents an individual microcapsule for HSA. Error bars are of the size of the symbol.

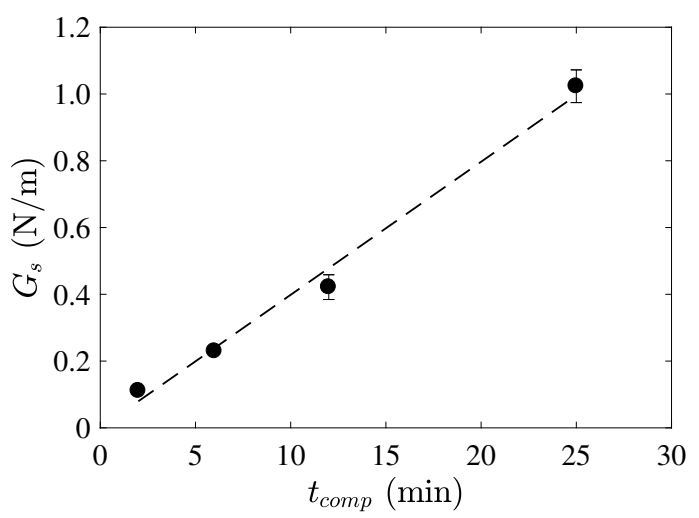

Fig. 6 Surface shear elastic modulus $G_{s}$ as a function of the complexation time $t_{\text {comp }} .0 .25 \% \mathrm{w} / \mathrm{v}$ chitosan, $0.36 \% \mathrm{w} / \mathrm{w}$ PFacidYN and $102 \pm 1 \mu \mathrm{m}$ radius. Error bars are the standard deviations.

method have the same elastic properties of membrane for a given formulation. The slope of the curve $D_{\infty}$ versus $\sigma$ provided us a constitutive rheological parameter, the surface shear elastic modulus $G_{s}$, that has been determined for each capsule (Equation 3 ). According to the formulation, $G_{s}$ ranged from $25 \mathrm{mN} / \mathrm{m}$ to $1.02 \mathrm{~N} / \mathrm{m}$ (Table 2 in supplementary materials). Each value of $G_{s}$ was the mean of 4 to 7 microcapsules. We estimated that the measurement uncertainty was within $\pm 6 \%$ with our set-up. For a given formulation, the standard deviation was also of this order. Our microfluidic procedure of fabrication and collection of suspensions of microcapsules allowed us to obtain not only monodisperse microcapsules (Figure 1), but also uniform elastic properties of the membrane of microcapsules.

It was also noteworthy that elastic properties of chitosan / PFacidYN microcapsules were independent of their radius. For example, human serum albumin (HSA) microcapsules fabricated from batch emulsification of two immiscible phases are polydisperse in size $\mathrm{e}^{46}$ and present variations of their elasticity of several 

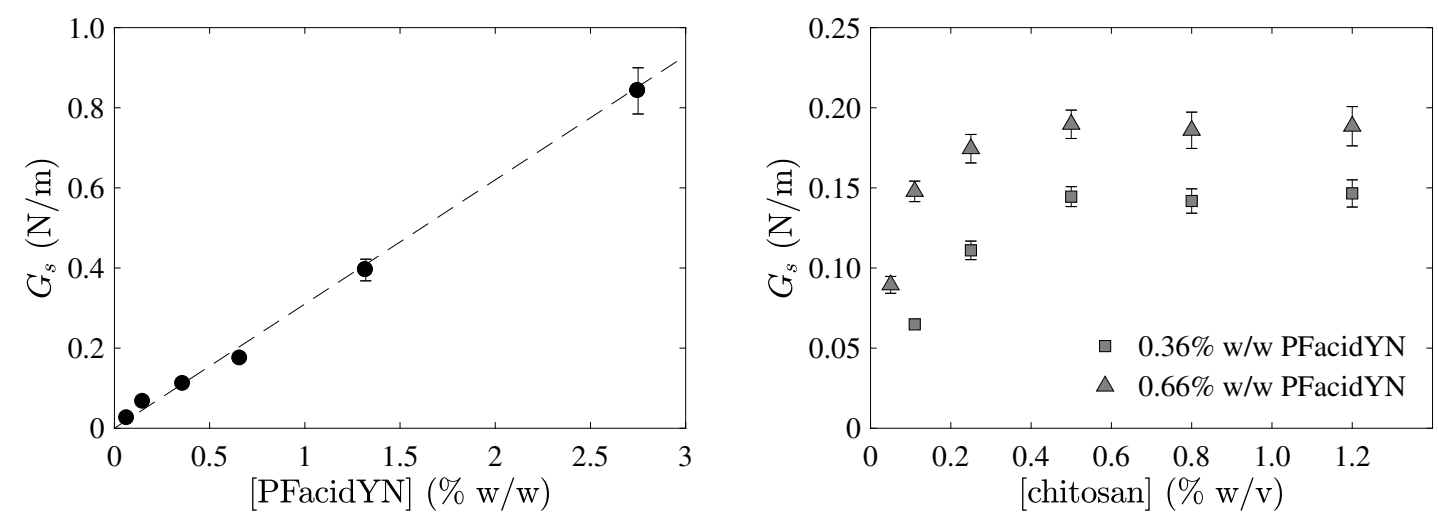

Fig. 7 Surface shear elastic modulus $G_{s}$ as a function of concentrion of complexing molecules. Top: $G_{s}$ increases linearly with the concentration of PFacidYN. $0.25 \% \mathrm{w} / \mathrm{v}$ chitosan, 2 min complexation time, $102 \pm 1 \mu \mathrm{m}$ radius. Bottom: $G_{s}$ saturates when the concentration of chitosan reaches a threshold of $0.5 \% \mathrm{w} / \mathrm{v} .0 .36 \% \mathrm{w} / \mathrm{w}$ and $0.66 \% \mathrm{w} / \mathrm{w}$ PFacidYN, 2 min complexation time, $102 \pm 1 \mu \mathrm{m}$ radius. Error bars are the standard deviations.

orders of magnitude 38,47 (Figure 5). For microcapsules prepared with $0.25 \% \mathrm{w} / \mathrm{v}$ chitosan solution, $0.36 \% \mathrm{w} / \mathrm{w}$ PFacidYN and a complexation time of 2 minutes, the mean $G_{s}$ was about 0.11 $\mathrm{N} / \mathrm{m}$ (mean of 60 microcapsules) with a standard deviation of \pm $0.02 \mathrm{~N} / \mathrm{m}$ for radii ranging from 80 to $200 \mu \mathrm{m}$. On the contrary, a factor 3.5 in size gave a factor 2000 in $G_{s}$ for HSA microcapsules. These strong differences can be explained by the kinetics of membrane formation. For chitosan / PFacidYN capsules, the thickness of the membrane formation scaled with the time at power $1 / 2$ on time scale of several hours in agreement with a growth by diffusion-controlled self-assembly of surfactant and/or chitosan chains ${ }^{18,48}$, while most of HSA molecules have been adsorbed at the water / oil interface and have reacted in $30 \mathrm{~min}^{49}$. Consequently, the maximum concentration of HSA molecules at the interface should be equal to $R[H S A] / 3$ in first approximation. The maximum surface concentration in HSA molecules thus increased both with the capsule size and with the initial bulk HSA concentration $^{38,47}$. This result enables us to fabricate microcapsules with uniform interfacial rheological properties that do not depend on the size.

A first way to control the interfacial rheological properties of the membrane of chitosan / PFacidYN microcapsules is to vary the time of complexation between both molecules. For fixed concentration of chitosan $(0.25 \% \mathrm{w} / \mathrm{v})$ and PFacidYN $(0.36 \% \mathrm{w} / \mathrm{w})$, the surface shear elastic modulus $G_{s}$ increased linearly with the complexation time $t_{\text {comp }}, G_{s} \simeq 0.04 t_{\text {comp }}$ (Figure 6). For a complexation time of 2 and 25 minutes, $G_{s}$ was $0.111 \pm 0.006 \mathrm{~N} / \mathrm{m}$ and $1.023 \pm 0.049 \mathrm{~N} / \mathrm{m}$, respectively, either a factor 10 . This result is correlated to the fact that the membrane thickness increases also with the square-root of the time ${ }^{18}$.

Another way to control the interfacial rheological properties of these microcapsules is also to change the total concentration in complexing molecules. Similarly to the complexation time, $G_{S}$ increased linearly with the concentration of surfactant PFacidYN (Figure 7). For $0.25 \% \mathrm{w} / \mathrm{v}$ chitosan and 2 minutes complexation of membrane, $G_{s}$ ranged from $0.025 \pm 0.001$ to $0.842 \pm 0.058$ $\mathrm{N} / \mathrm{m}$ when the concentration of PFacidYN increased from 0.066 to $2.75 \% \mathrm{w} / \mathrm{w}$. In this case, the linear relationship between $G_{S}$ and the concentration of PFacidYN was $G_{s} \simeq 0.31[$ PFacidYN]. For lower concentration of surfactant, microcapsules coalesced easily which were prevented to explore in this study. This linear evolution of $G_{s}$ was correlated to an increase of the membrane thickness that has been measured by AFM. Measuring the topographies of collapsed microcapsules (Figure 2) allowed us to determine the magnitudes of the membrane thickness for different concentrations of PFacidYN. We found that the thickness of the membrane increased from about $40-50 \mathrm{~nm}$ up to $700-950 \mathrm{~nm}$ for concentrations of PFacidYN of 0.066 and $2.75 \% \mathrm{w} / \mathrm{w}$ respectively. At intermediate concentrations of surfactant $(0.66-1.32 \%$ $\mathrm{w} / \mathrm{w}$ PFacidYN), the membrane thickness was about $100-170 \mathrm{~nm}$. The uncertainty is relatively large as this kind of measurement is technically difficult due to poor adhesion of microcapsules on the substrate and the difficulty to determine the number of layers for each step. The uncertainty on the number of layers can be reduced if a region is scanned at the interface between the microcapsule and the substrate where there is more chance to have only two layers of membrane. Several measurements have been done on the same microcapsule, but these values have to be considered rather as an order of magnitude than an highly accurate measurement. However, the variations between the samples were sufficiently large to conclude. At this stage, it is not reasonable to conclude more precisely on the trend of the evolution of the membrane thickness with others formulation and process parameters. We can also deduce from Equation 4 that the Young modulus of the membranes increased slightly from 1.6 to $3.2 \mathrm{MPa}$ contrarily to $G_{s}$ that was multiplied by a factor 33 . This order of magnitude of Young modulus is equivalent to the one measured by AFM on HSA microcapsules ${ }^{38}$. It means that the variation of $G_{s}$ can be mainly attributed to the variation of the membrane thickness and the membrane structure is almost homogeneous.

The surfacic shear elastic modulus $G_{S}$ increases also with the concentration of chitosan up to reach a plateau value for a critical concentration, about $0.5 \% \mathrm{w} / \mathrm{v}$ (Figure 7). Above this critical concentration, there was so a sufficient quantity of chitosan molecules available to saturate the interface and the quantity of surfactant available for the complexation is the limiting factor, since the plateau value of $G_{s}$ depends on its concentration (Figure 7). 


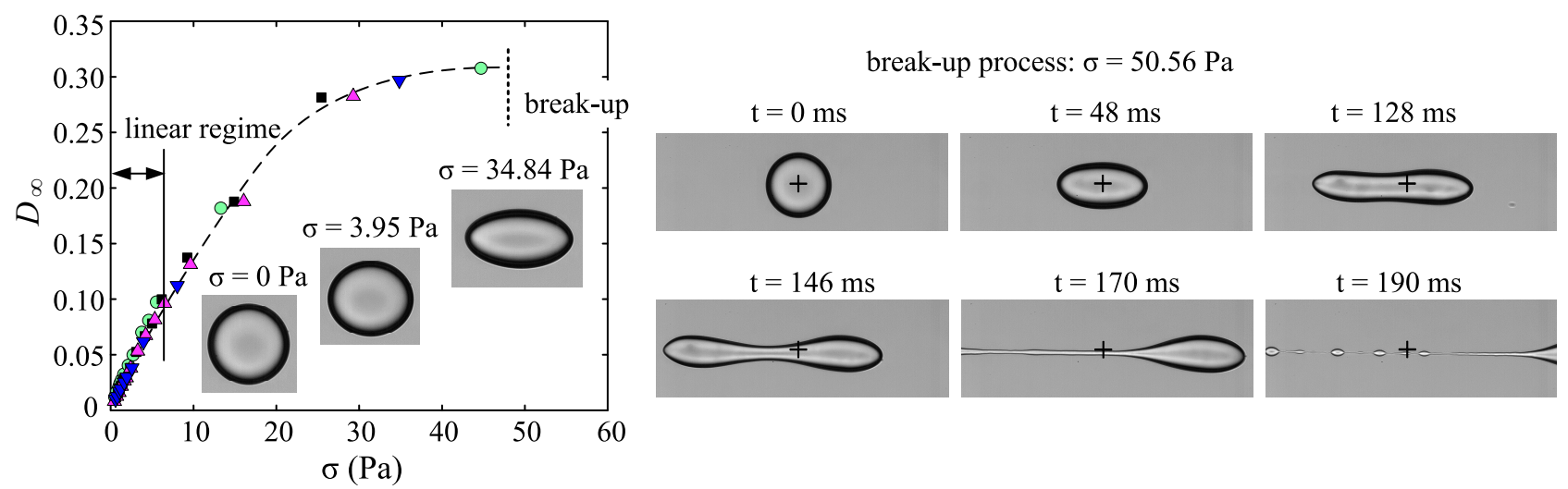

Fig. 8 Break-up of a chitosan / PFacidYN microcapsules in an elongation flow. Left: The steady-state Taylor parameter $D_{\infty}$ increases non-linearly with the hydrodynamic stress $\sigma$ above values of 0.1 . Images show the evolution of the steady-state shape. Each symbol corresponds to a microcapsule. Right: When the break-up threshold is exceed $(\sigma=50.56 \mathrm{~Pa})$, the microcapsule is stretched up to the formation of two lobs connected by a thin filament $(t=146 \mathrm{~ms})$ that is then destabilized in droplets $(t=190 \mathrm{~ms})$. The center of the flow chamber is indicated by $+.0 .25 \% \mathrm{w} / \mathrm{v}$ chitosan, $0.066 \% \mathrm{w} / \mathrm{w}$ PFacidYN, 2 min complexation time, $102 \pm 1 \mu \mathrm{m}$ radius. See Movie 2 in supplementary materials.

Finally, we show that the elasticity of self-assembling biopolymer microcapsules can be easily controlled by the microfluidics process of fabrication in a large way (factor 10). Moreover, this process allowed us to get monodisperse suspensions of microcapsules with uniform elasticity that can be kept stable outside the system. Tuning the various properties (e.g. size, morphology, adhesion, mechanics) of microcapsules combined with costefficient route of farbrication is still a challenge and should open numerous innovations as in biotechnology ${ }^{3}$. For example, Gunes et al. ${ }^{18}$ fabricated polyelectrolytes microcapsules with a flow-focusin microchip where the complexing molecules were dispersed separately in the oil and water phase according to their solubility, to avoid layer-by-layer construction. A similar system has been reproduced by Kaufman et al. ${ }^{50}$ and in the present paper. More sophisticated microfluidic devices have been developed to estimate the elastic modulus simultaneously with the production of microcapsules ${ }^{51-53}$. These set-ups are useful to determine the kinetic process at the early stage of membrane formation ${ }^{52}$, but they are limited by the testing time-scale of a few seconds, and cannot give access to the final properties of the microcapsules whereas the typical time to form a stable membrane is in order of a few minutes. Moreover, the stability of the suspension outside the generation serum is not guaranteed ${ }^{51}$, i.e. the resulting osmotic shock may have too large consequences. In contrast to the previous workers, we have deliberately chosen to uncouple the formation of monodisperse droplets with surfactant/chitosan adsorption to prevent coalescence from the real formation of the membrane, to be able to control the complexation time (from 2 to $25 \mathrm{~min}$ ), the growth of the membrane and therefore its elastic properties. The uncoupling fabrication of microcapsules with their mechanical characterization allowed us to guarantee the stability of the suspensions and also to measure a constitutive rheological parameter of the membrane, the surface elastic modulus, instead of a deformation under specific flow conditions making difficult to determine constitutive rheological parameters without numerical simulations $^{51}$. Furthermore, high shear stress can be generated with the elongation flow chamber that have access to the non- linear interfacial rheological properties of chitosan/PFacidYN microcapsules.

\subsection{Break-up, yield stress and plasticity of microcapsules}

In the regime of large deformation (typically $D_{\infty}>0.1$ ), the nonlinear mechanical behavior of chitosan / PFacidYN microcapsules can also be tuned by the formulation (concentration, time) to obtain microcapsules that can present a yield stress associated to plastic deformations and/or that burst easily under an applied hydrodynamic stress. Few examples of these behaviors are presented in a quantitative way.

Chitosan / PFacidYN microcapsules with the thinnest membranes $(<100 \mathrm{~nm})$ can be easily bursted under hydrodynamic stress with our elongation flow chamber (Figure 8-left). Beyond the small deformation regime, the evolution of the steady-state deformation tended to slow-down with the stress. The maximum steady deformation $D_{\infty}$ was about $0.3\left(L_{\infty} / R=1.3\right)$ for a stress of $36 \mathrm{~Pa}$ without membrane fracture $(0.25 \% \mathrm{w} / \mathrm{v}$ chitosan, $0.066 \%$ $\mathrm{w} / \mathrm{w}$ PFacidYN and 2 min complexation time). In this example, the deformation was elastic as microcapsules recovered their initial shapes at rest and stress larger than 40 50 Pa were required to burst the microcapsule. The critical stress increased also with $G_{s}$.

The process of break-up of chitosan / PFacidYN microcapsules occurred in the time scale of milliseconds (Figure 8-right). Whereas the long axis $L$ increased with time, a pinch appeared at the center of the ellipsoidal microcapsule. The shape was unstationary and evolved to a dumbbell with a stronger central pinching due to the right and left lobs which stretched the capsule, reminiscent of recent numerical simulations ${ }^{54}$. Finally, a thin filament connected the two opposite lobs that moved away (see movie in supplementary materials). Contrary to Newtonian droplets, we have not been able in this study to obtain long and quite large threads. The elastic response of membrane protected efficiently the integrity of large lobs and prevented a leak of liquid from the lobs to the thread. This thin filament was unstable as expected from the Plateau-Rayleigh instability driven by the 


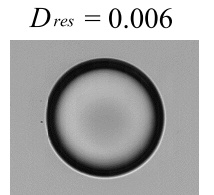

$\sigma=9.46 \mathrm{~Pa}$

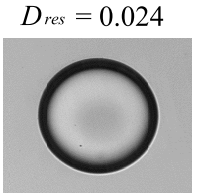

$\sigma=89.32 \mathrm{~Pa}$

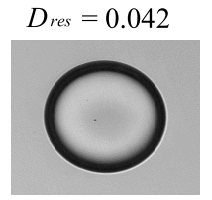

$\sigma=127.26 \mathrm{~Pa}$

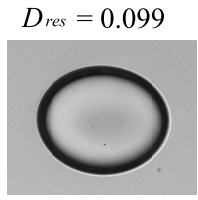

$\sigma=171.60 \mathrm{~Pa}$

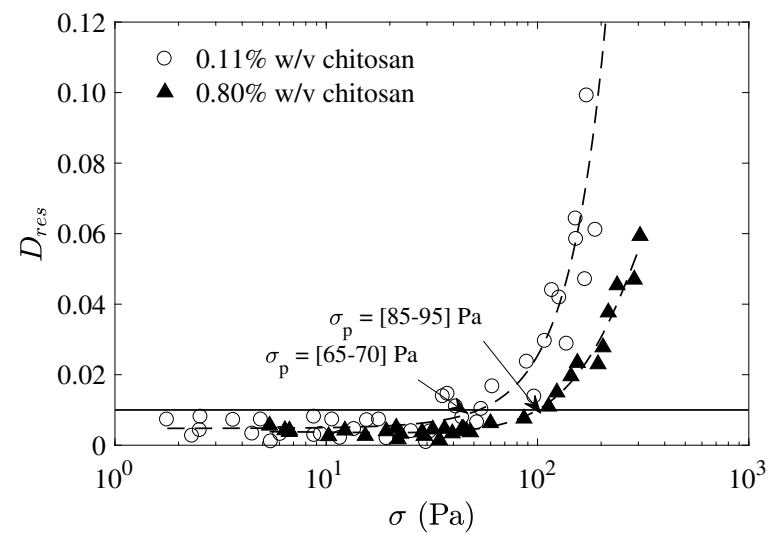

Fig. 9 Plastic deformations of chitosan / PFacidYN microcapsules. Top: The residual Taylor parameter $D_{\text {res }}$ increases with the hydrodynamic stress $\sigma$ just after stopping the elongation flow (within $0.5 \mathrm{~min}$ ). Bottom: The yield stress demarcates elastic and plastic deformation $\sigma_{p}$ is defined by $D_{\text {res }}>0.01$ and depends on the formulation of microcapsules. Lines are guides for the eyes. $0.36 \% \mathrm{w} / \mathrm{w}$ PFacidYN, 2 min complexation time, $102 \pm 1 \mu \mathrm{m}$ radius.

surface tension of fluid interfaces ${ }^{55-57}$ which generated a pattern of pearls along the filament. These observations question the existence of a possible solid to liquid transition of the membrane at these rate of deformations that could be studied on plane interfaces by double-wall ring geometries ${ }^{25}$. Moreover, in elongation flow, droplets with surfactant also exhibited tip-streaming 58 which is not the case here at least in the range of studied parameters.

For the thickest Chitosan / PFacidYN membranes (> $200 \mathrm{~nm}$ ), burst of microcapsules was not observed in the range of hydrodynamic stress available with our set-up. However, plasticity and relaxation times of few minutes were observed. The Taylor parameter at rest of microcapsules has been measured after applying different levels of hydrodynamic stress in interval of time between stopping the flow and measurement of $0.5 \sim 1$ min (Figure 9). In regime of small deformations there were no residual deformation that can be detected by our visualization method $\left(D_{\text {res }}<\right.$ 0.008). However, the capsules that were exposed to larger stress did not return immediately to their spherical shape at rest. The residual deformation $D_{\text {res }}$ increased non-linearly with the value of applied stress on the microcapsules up to 0.1. The critical stress that separates elastic and plastic deformations can be quantified and varied with the formulation. From these observations, we cannot deduce the yield stress of the membrane. In fact, the interface being closed, the state of stress in the membrane is inhomogeneous when the capsule is deformed. Previous numerical simulations have shown that tension is maximal at the tips of the capsule $^{59}$. We can so deduce that plastic deformations are probably localized near the tips of the deformed capsule.
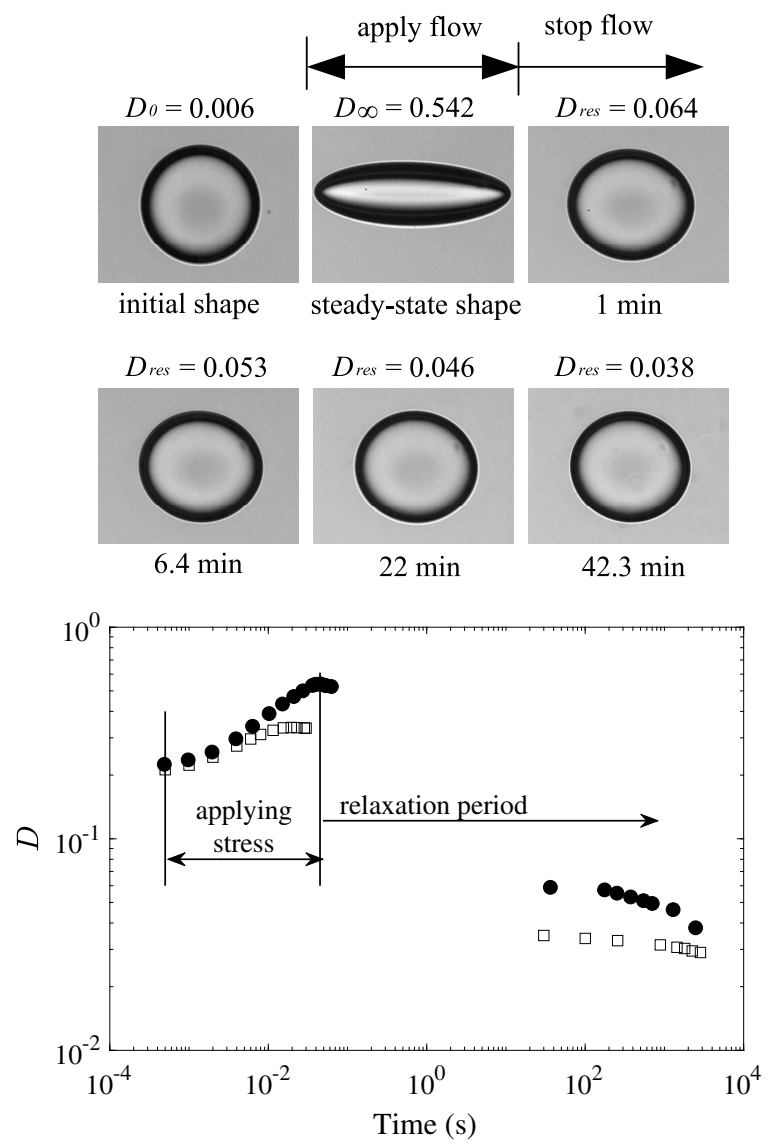

Fig. 10 Relaxation of the residual deformation. Top: A microcapsule that is spherical at rest is deformed up to $D_{\infty}$ of 0.54 . Just after the flow (1 min), a residual deformation $D_{\text {res }}$ remains at rest and decreases slowly with the time. Bottom: Taylor parameter $D$ as a function of the time. The first and second parts of the curves correspond to the elongationnal flow and to the period at rest respectively. Microcapsules do not recover their initial shape (i.e. $D_{\text {res }}>0.01$ on the timescale of the observation (about $45 \mathrm{~min}$ ). Circles: $0.11 \% \mathrm{w} / \mathrm{v}$ chitosan, $0.36 \% \mathrm{w} / \mathrm{w}$ PFacidYN, 2 min complexation time, $G_{s}=0.065 \mathrm{~N} / \mathrm{m}, \sigma=153 \mathrm{~Pa}$. Squares: $0.25 \% \mathrm{w} / \mathrm{v}$ chitosan, $0.36 \% \mathrm{w} / \mathrm{w}$ PFacidYN, 6 min complexation, $G_{s}=0.230 \mathrm{~N} / \mathrm{m}, \sigma=744 \mathrm{~Pa}$.

After the capsule has been highly deformed and the flow was stopped, we observed two phases of shape relaxation (Figure 10). In only few seconds, the deformation was much lower than the steady-state deformation $D_{\infty}$. On time scales of few minutes, the shape relaxed slowly. Microcapsules with large steady-state deformations had larger residual deformations, and seemed to require longer relaxation times (up to $40 \mathrm{~min}$ ). Due to experimental limitations (e.g., sedimentation of capsules and working length of objective lens), longer relaxation time was not exerted.

In summary, extensional flow set-up allows us to exert large hydrodynamic stresses on the chitosan / PFacidYN microcapsules, for which deviation from the linear elasticity was observed, contrarily to most common methods like AFM or osmotic pressure test $^{12}$. This method give insights for the first time on the nonlinear mechanical behavior of a kind polyelectrolyte microcapsules. Microcapsules presented remarkably non-linear behaviors, such as permanent deformation and membrane break-up. As the state of tension on the membrane is inhomogeneous, experiments 
on plane interfaces with double-wall ring geometries should be relevant to determine solid-liquid transitions of these kind of membranes ${ }^{18,25}$. Chitosan / PFacidYN microcapsules stand out also of HSA capsules that can be stretched up to $180 \%$ of elongation without plasticity or break-up. ${ }^{40}$ The difference between the two systems is the nature of bonds : electrostatic versus cross-linking. Chang and Olbricht ${ }^{32,33}$ probably first conducted the study of the residual deformation and membrane break-up of nylon capsules with a size order of $2 \sim 4 \mathrm{~mm}$ under an elongation flow and Couette flow. Other studies handled with the polysiloxane capsules ${ }^{37,60}$, with which permanent deformation and membrane fracture were also observed. For chitosan/PFacidYN microcapsules, probably it is also interesting to see that these different behaviors can be obtained with the same complexing molecules by varying the membrane thickness. It has also been shown by Kaufman et al. ${ }^{61}$ that complexing the polyelectrolytes with nanoparticles is a lever to control the plastic deformations for membranes of a few micrometers thick.

\section{Conclusion}

Self-assembling and self-healing ${ }^{16,18}$ biopolymer chitosan/PFacidYN microcapsules exhibit a variety of linear and non-linear interfacial rheological properties. In the framework of interfacial properties of capsule membranes, this study generalizes the relevance of the extensional flow method $21,40,42$ to probe the full scale of deformations of different kinds of microcapsules, whatever the cohesive strength of membrane materials from strong with crosslinking of HSA for example ${ }^{38}$ to weak as shown in this study on polyelectrolyte multilayers.

If microfluidics is a way to produce monodisperse suspensions of droplets with a small standard deviation, a crucial step to make uniform suspensions, it does not prevent from having a larger deviation in interfacial rheological properties of capsule membranes. We have shown that combining the microfluidic tools with a cost-effective physico-chemical process involving a singlestep interfacial complexation of polyelectrolytes allows to generate various uniform suspensions of microcapsules. Indeed, it is remarkable to observe that with the same complexing molecules, the interfacial rheological properties can be tuned and various degrees of elasticity, yield stress, plasticity and break-up can be obtained. The surface elastic modulus ranged from $25 \mathrm{mN} / \mathrm{m}$ to $1 \mathrm{~N} / \mathrm{m}$ and was correlated to the thickness of the membrane (40 - $950 \mathrm{~nm}$ ). This wealth of mechanical behavior can so be mainly attributed to the variation of the membrane thickness, as the order of magnitude of the Young modulus was almost constant whatever the concentration of complexing molecules was (1.6 $3 \mathrm{MPa}$, Eq. 4). As measurements have been realized in steadystate, the interfacial viscosity of these microcapsules was not determined. Experiments in shear flows are more suitable to determine this parameter by tracking the motion of the membrane with microparticles ${ }^{41}$, but the complexity is also to choose the accurate visco-elastic model ${ }^{22,62}$. Beyond the interests of microencapsulation in various domains of engineering, these objects can also be used as mastered model systems of red blood cells for micro-hydrodynamics experiments ${ }^{63}$. Finally, this paper is a step forward to 'engineer' the interfacial rheology of microcapsules for specific purposes.

\section{Acknowledgements}

This work has benefited from financial support from the ANR Polytransflow (ANR-13-BS09-0015), from Labex MEC (ANR-11LABX-0092) and from CNES. Also, K. Xie acknowledges the China Scholarship Council (CSC 201504490090) to fund his PhD scholarship. F. Dubreuil ackowledges the Nanobio-ICMG plateform (FR 2607) for the AFM facilities. The authors thank Cécile Soubeyrand from IMCD group for the gift of Wacker AP 1000 and Laurent Eymeoud from Plasgaard for the gift of lecithinYN (Palsgaard 4448, E442).

\section{Supplementary Materials}

The following files are available free of charge.

- Movie 1: Deformation of a chitosan / PfacidYN microcapsule in the regime of large deformations.

- Movie 2: Break-up process of a chitosan / PfacidYN microcapsule.

- Tables 1 \& 2: Formulations of microcapsules studied and surface shear elastic modulus $G_{s}$.

\section{References}

1 M. A. Trojer, L. Nordstierna, J. Bergek, H. Blanck, K. Holmberg and M. Nyden, Adv. Colloid Interface Sci., 2015, 222, 18-43.

2 S. Anandhakumar, V. Nagaraja and A. M. Raichur, Colloids Surf., B, 2010, 78, 266-74.

3 A. Choi, K. D. Seo, B. C. Kim and D. S. Kim, Lab Chip, 2017, 17, 591-613.

4 D. J. McClements, J. Food Sci., 2015, 80, N1602-N1611.

5 L. Bosnea, T. Moschakis and C. Biliaderis, Food Funct., 2017, 8, 554-562.

6 R. Hejazi and M. Amiji, J. Controlled Release, 2003, 89, 151165.

7 Y. Long, C. Liu, B. Zhao, K. Song, G. Yang and C.-H. Tung, NPG Asia Mat., 2014, 7, e148.

8 D. Langevin, Adv. Colloid Interface Sci., 2009, 147, 170-177.

9 C. Bain, P. M. Claesson, D. Langevin, R. Meszaros, T. Nylander, C. Stubenrauch, S. Titmuss and R. Von Klitzing, Adv. Colloid Interface Sci., 2010, 155, 32-49.

10 J. J. Richardson, J. Cui, M. Björnmalm, J. A. Braunger, H. Ejima and F. Caruso, Chemical Reviews, 2016, 116, 1482814867.

11 F. Dubreuil, N. Elsner and A. Fery, Eur. Phys. J. E: Soft Matter Biol. Phys., 2003, 12, 215-221.

12 A. Fery, F. Dubreuil and H. Möhwald, New journal of Physics, 2004, 6, 18.

13 D. Grigoriev, T. Bukreeva, H. Möhwald and D. Shchukin, Langmuir, 2008, 24, 999-1004.

14 S. Le Tirilly, C. Tregouët, S. Bône, C. Geffroy, G. Fuller, N. Pantoustier, P. Perrin and C. Monteux, ACS Macro Letters, 2014, 4, 25-29. 
15 L. Chiappisi and M. Gradzielski, Adv. Colloid Interface Sci., 2015, 220, 92-107.

16 J. D. de Baubigny, C. Trégouët, T. Salez, N. Pantoustier, P. Perrin, M. Reyssat and C. Monteux, Scientific Reports, 2017, 7, 1265.

17 A. Sharipova, S. Aidarova, D. Grigoriev, B. Mutalieva, G. Madibekova, A. Tleuova and R. Miller, Colloids and Surfaces B: Biointerfaces, 2016, 137, 152-157.

18 D. Z. Gunes, M. Pouzot, M. Rouvet, S. Ulrich and R. Mezzenga, Soft Matter, 2011, 7, 9206-9215.

19 M. G. Peter, Chitin and Chitosan in Fungi, Wiley Online, 6th edn, 2005.

20 D. Barthes-Biesel and H. Sgaier, J. Fluid Mech., 1985, 160, 119-135.

21 P. Dimitrakopoulos, J. Fluid Mech., 2014, 745, 487-508.

22 A. Yazdani and P. Bagchi, J. Fluid Mech., 2013, 718, 569-595.

23 P. Erni, P. Fischer, E. J. Windhab, V. Kusnezov, H. Stettin and J. Lauger, Rev. Sci. Instrum., 2003, 74, 4916-4924.

$24 \mathrm{H}$. Brenner, Interfacial transport processes and rheology, Elsevier, 2013.

25 G. G. Fuller and J. Vermant, Annu. Rev. Chem. Biomol. Eng., 2012, 3, 519-543.

26 A. Fery and R. Weinkamer, Polymer, 2007, 48, 7221-7235.

27 J. Van Raamsdonk and P. Chang, J. Biomed. Mater. Res., 2001, 54, 264-271.

28 L. Rolland, E. Santanach-Carreras, T. Delmas, J. Bibette and N. Bremond, Soft matter, 2014, 10, 9668-9674.

29 C. Gao, E. Donath, S. Moya, V. Dudnik and H. Möhwald, Eur. Phys. J. E: Soft Matter Biol. Phys., 2001, 5, 21-27.

30 B. Sarrazin, N. Tsapis, L. Mousnier, N. Taulier, W. Urbach and P. Guenoun, Langmuir, 2016, 32, 4610-4618.

31 S. Knoche, D. Vella, E. Aumaitre, P. Degen, H. Rehage, P. Cicuta and J. Kierfeld, Langmuir, 2013, 29, 12463-12471.

32 K. S. Chang and W. L. Olbricht, J. Fluid Mech., 1993, 250, 587-608.

33 K. S. Chang and W. L. Olbricht, J. Fluid Mech., 1993, 250, 609-633.

34 G. Pieper, H. Rehage and D. Barthes-Biesel, J. Colloid Interface Sci., 1998, 202, 293-300.

35 A. Walter, H. Rehage and H.Leonhard, Colloid Polym. Sci., 2000, 278, 169-175.

36 A. Unverfehrt, I. Koleva and H. Rehage, J. Phys.: Conf. Series, 2015, 602, 012002.

37 I. Koleva and H. Rehage, Soft Matter, 2012, 8, 3681.

38 C. de Loubens, J. Deschamps, M. Georgelin, A. Charrier, F. Edwards-Lévy and M. Leonétti, Soft Matter, 2014, 10, 4561-4568.

39 E. Lac, D. Barthès-Biesel, N. Pelekasis and J. Tsamopoulos, J. Fluid Mech., 2004, 516, 303-334.

40 C. de Loubens, J. Deschamps, G. Boedec and M. Leonetti, J. Fluid Mech., 2015, 767, R3.

41 C. de Loubens, J. Deschamps, F. Edwards-Levy and M. Leonetti, J. Fluid Mech., 2016, 789, 750-767.
42 S. Haward, Biomicrofluidics, 2016, 10, 043401.

43 Minifie, Chocolate, Cocoa and Confectionary: Science and Technology, Aspen, 1989.

44 T. Glawdel, C. Elbuken and C. L. Ren, Phys. Rev. E: Stat., Nonlinear, Soft Matter Phys., 2012, 85, 016322.

45 D. Barthes-Biesel and J. Rallison, J. Fluid Mech., 1981, 113, 251-267.

46 F. Edwards-Levy, in Serum Albumin: Structure, Functions and Health Impact, Nova Science, 2011, ch. Microparticulate Drug Delivery Systems Based on Serum Albumin, pp. 69-100.

47 J. Gubspun, P.-Y. Gires, C. De Loubens, D. Barthes-Biesel, J. Deschamps, M. Georgelin, M. Leonetti, E. Leclerc, F. Edwards-Levy and A.-V. Salsac, Colloid Polym. Sci., 2016, 294, 1381-1389.

48 M. Rinaudo, N. Kil'deeva and V. Babak, Russ. J. Gen. Chem., 2008, 78, 2239-2246.

49 M. C. Andry, F. Edwards-Levy and M. C. Levy, Int. J. Appl. Pharm., 1996, 128, 197-202.

50 G. Kaufman, R. Boltyanskiy, S. Nejati, A. R. Thiam, M. Loewenberg, E. R. Dufresne and C. O. Osuji, Lab Chip, 2014, 14, 3494-3497.

51 T. Chu, A.-V. Salsac, D. Barthes-Biesel, L. Griscom, F. Edwards-Levy and E. Leclerc, Microfluid. Nanofluid., 2013, 14, 309-317.

52 I. Polenz, Q. Brosseau and J.-C. Baret, Soft matter, 2015, 11, 2916-2923.

53 N. Galle and V. Steinberg, Microfluid. Nanofluid., 2016, 20, 156.

54 P. Dimitrakopoulos, Physical Review Fluids, 2017, 2, 063101.

55 L. Rayleigh, Proc. London Math. Soc., 1878, 1, 4-13.

56 S. Tomotika, Proc. R. Soc. London, Ser. A, 1935, 150, 322-337.

57 G. Boedec, M. Jaeger and M. Leonetti, J. Fluid Mech., 2014, 743, 262-279.

58 G. Taylor, Proc. R. Soc. London, Ser. A, 1934, 146, 501-523.

59 W. Dodson III and P. Dimitrakopoulos, Physical review letters, 2008, 101, 208102.

60 A. Walter, H. Rehage and H. Leonhard, Colloids Surf., A, 2001, 183, 123-132.

61 G. Kaufman, S. Nejati, R. Sarfati, R. Boltyanskiy, M. Loewenberg, E. R. Dufresne and C. O. Osuji, Soft matter, 2015, 11, 7478-7482.

62 J. Gounley, G. Boedec, M. Jaeger and M. Leonetti, J. Fluid Mech., 2016, 791, 464-494.

63 P. M. Vlahovska, D. Barthès-Biesel and C. Misbah, C. R. Phys., 2013, 14, 451-458. 